\title{
Do Herbal Formulas Influence the International Normalized Ratio of Patients Taking Warfarin? A Retrospective Study
}

\author{
Hsu-Yuan Lu, Seung-Yeon Cho, Seong-Uk Park, Woo-Sang Jung, \\ Sang-Kwan Moon, Jung-Mi Park, and Chang-Nam Ko \\ Department of Cardiology and Neurology, College of Korean Medicine, Kyung Hee University, No. 26 Kyungheedae-ro, \\ Dongdaemun-gu, Seoul 130-872, Republic of Korea
}

Correspondence should be addressed to Chang-Nam Ko; kcn202@khu.ac.kr

Received 23 December 2014; Accepted 5 February 2015

Academic Editor: Waris Qidwai

Copyright (C) 2015 Hsu-Yuan Lu et al. This is an open access article distributed under the Creative Commons Attribution License, which permits unrestricted use, distribution, and reproduction in any medium, provided the original work is properly cited.

\begin{abstract}
Warfarin is a common anticoagulant agent for cardiovascular diseases, and it is known to interact with several foods and drugs. Several studies report an interaction between warfarin and herbal medicines; however, the influence of herbal medicines on the international normalized ratio (INR) is still controversial. We investigated the influence of herbal formulas on INR of patients taking warfarin. We searched electronic medical records of inpatients for INR results. Then, we compared the changes in INR and any adverse events between the group taking herbal formulas and warfarin (herbal group) and another group taking warfarin only (nonherbal group). Eighty-six patients were included; 45 patients were assigned to the herbal group and 41 patients to the nonherbal group. The herbal group had taken the same dose of warfarin for a longer period. The nonherbal group had a slightly higher mean INR value than the herbal group. The ratio of INR less than 2 and greater than 3 , the ratio of INR that increased or decreased by one or more compared to the initial INR, and the ratio of adverse events were not significantly different between the two groups. It is suggested that use of herbal formulas may not influence INR value.
\end{abstract}

\section{Introduction}

Warfarin is a common anticoagulant agent that is used in various cardiovascular diseases, venous thrombosis, prevention of systematic embolism, atrial fibrillation, and cardiac valve diseases [1]. It is known to interact with several drugs and foods to influence the international normalized ratio (INR) [2]. Herbal formulas have been used for several thousands of years in China, Korea, and Japan; in recent years, many Western countries have started using herbal formulas [3]. As many countries use herbal formulas, the potential effects of these herbal formulas on the INR have been reported [4-6]. In one report, an herbal formula combination of Tribulus terrestris, Avena sativa, and Panax ginseng was reported to result in a sudden increase in the INR of patients who took warfarin for aortic valve replacement or atrial fibrillation [4]. However, another randomized controlled trial study reported that Panax ginseng did not have any effect on INR [7]. Whether or not herbal formulas influence INR is still controversial.
Several previous researchers have reported interactions between herbal formulas and warfarin, but there are few citations published in Science Citation Index (SCI) journals, and there are some important limitations in all of the studies [815]. Choi et al. investigated the interaction between herbal formulas and warfarin in 27 patients using the World Health Organization-The Uppsala Monitoring Centre causality categories and modified drug interaction probability scale (mDIP scale). However, the study could not clearly clarify interactions between herbal formulas and warfarin because the mDIP scale only measures single components and is not suitable for complex formulas. Kim et al. [9], Lee et al. [10], Kim et al. [11], and Lee and Ryu [12] investigated patients taking warfarin who were hospitalized at a traditional Korean Medicine hospital. Kim et al. [9] reported that herbal formulas may slightly affect INR, but other studies reported that herbal formulas did not affect INR [10-12]. Kwon et al. [13] and Jung et al. [14] investigated the mean INR of patients taking warfarin and found no statistically significant differences between INR before and INR during the use of the 
herbal formulas. The results by Jung et al. [14] are limited because they only investigated the mean INR; the data does not show the change in INR in the individual subjects as a result of the herbal formulas. In another study, Kwon et al. [15] investigated 28 patients who took Panax ginseng in conjunction with warfarin. Because the study only investigated herbal formulas that included Panax ginseng, it was difficult to apply the findings to other herbal formulas that do not contain Panax ginseng.

In this study, we compared a group taking herbal formulas in conjunction with warfarin (herbal group) and another group taking warfarin only (nonherbal group) in order to investigate whether herbal formulas affect INR.

\section{Materials and Methods}

The study was performed in accordance with the ethical standards of the Helsinki Declaration. The Institutional Review Board of Kyung Hee University Hospital at Gangdong approved this study (KHNMC-OH-IRB 2013-015).

We searched electronic medical records of patients hospitalized from June 2006 to May 2013 at the Department of Korean Medicine or the Department of Physical Medicine and Rehabilitation in Stroke and Neurological Disorders Center, Kyung Hee University Hospital, at Gangdong to find patients who satisfied our criteria: (1) 18 years of age or older, (2) previously taking warfarin before hospitalization and taking the same dose of warfarin for at least five days since the day of hospitalization, (3) initial INR in the range of 2 to 3 , and (4) follow-up measurements conducted once or more during hospitalization. In order to exclude the possible effects on INR, any patients that had taken any herbal medicine in the 30 days before hospitalization were excluded. If the patients were hospitalized more than twice, we regarded each hospitalization as an individual subject. In addition, we designated patients who took herbal formulas with warfarin as the herbal group and those who took only warfarin as the nonherbal group. We then investigated the following items for up to 30 days: (1) ratio of INR less than two and greater than three, (2) ratio of INR changed by one or more from the initial INR, (3) mean INR, and (4) ratio and types of adverse events. If warfarin dose was changed, a patient was discharged in either group, or a patient in the nonherbal group started taking an herbal formula, we investigated INR and adverse events up to the time of that change.

Herbal formulas were prescribed decoctions or extracts. Herbal medicines for decoctions were purchased from Kyung Hee Herb Pharm Co. (Seoul, Korea), and extracts were purchased from the following companies: Kyung Hee Herb Pharm Co. (Seoul, Korea), Tsumura Co., Ltd. (Tokyo, Japan), Hamsoa Pharmaceutical Co., Ltd. (Seoul, Korea), Kwang Dong Pharmaceutical Co., Ltd. (Seoul, Korea), Jeil Hanbang Co., Ltd. (Seoul, Korea), Hankook Shinyak Pharm Co., Ltd. (Chungcheongnam-do, Korea), and Kiwha Bio Co., Ltd. (Gyeongsangnam-do, Korea). Decoctions were prepared using two or more herbal ingredients in 1.2 to 1.5 liters of water boiled in decoction vessels at greater than $100^{\circ} \mathrm{C}$ for $2 \mathrm{hrs} 30 \mathrm{~min}$. All patients in the herbal group took herbal decoctions of $50 \mathrm{~mL}$ to $120 \mathrm{~mL}, 2$ hrs after every meal, and took extracts of 6-18 $\mathrm{g}$ according to symptoms, if needed.
2.1. Statistical Analyses. Data are expressed as number or mean \pm standard deviation. Categorical variables were analyzed by Pearson's Chi-square test, and continuous variables were analyzed by Student's $t$-test. $P$ values less than 0.05 were considered significant. All data were analyzed using SPSS for Windows, version 18.0 (Statistical Package for the Social Sciences Inc., Chicago, IL, USA).

\section{Results}

Eighty-six patients were included; 45 patients were assigned to the herbal group and 41 patients were assigned to the nonherbal group. The herbal group took the same dose of warfarin for a longer period $(P=0.027)$, had more cerebral infarction as an indicator of warfarin use $(P=0.036)$, and showed a higher hemoglobin count $(P=0.045)$ compared to the nonherbal group. There were no statistically significant differences in other baseline characteristics between the two groups (Table 1).

There were no statistically significant differences in the ratio of INR less than 2 or greater than 3 or the ratio of increase/decrease of more than one from the initial INR between the two groups. However, the nonherbal group had a slightly higher mean INR value than the herbal group (Table 2).

No major bleeding was reported in any patients, but there were some minor bleeding events in both groups. In the herbal group, one patient appeared to have hemoptysis during tracheostomy suctioning, one showed hematuria, and one patient showed melena. In the nonherbal group, one patient complained of hematuria, and another patient showed hemoptysis and subconjunctival hemorrhage. None of these adverse events were fatal, and all patients showed recovery. There was no statistically significant difference in the adverse events ratio between the two groups (Table 3).

\section{Discussion}

Warfarin is an antagonist of vitamin $\mathrm{K}$ and is known to interact with several foods and drugs [2]. Because of its narrowing therapeutic index, patients require consistent monitoring when taking warfarin [16]. Several studies have reported that herbal formulas affect INR; however, there were limits to the research methods used in these studies, so the issue is still controversial [7-15]. In this study, we found no substantial differences between the herbal and the nonherbal groups with regard to the ratio of INR less than two and greater than three or the ratio of INR value changed by one or more units from the initial INR. Some previous studies reported that herbal medicines could affect INR $[17,18]$; in these cases, the herbal medicines were administered by the patients themselves and were not prescribed by experts. In contrast, some randomized controlled trials reported that herbal formulas prescribed by experts did not affect INR [10-14]. Similar results were shown in our study; therefore, we concluded that even if some patients took herbal formulas that contain Panax ginseng, Dong quai, and other medicines that can affect INR [19], the influence may be prevented when the herbal formulas 
TABLE 1: Baseline characteristics between the herbal and nonherbal groups.

\begin{tabular}{|c|c|c|c|}
\hline & Herbal group $(n=45)$ & Nonherbal group $(n=41)$ & $P$ value \\
\hline Male/female & $19 / 26$ & $17 / 24$ & 0.943 \\
\hline Age (year) & $70.0 \pm 12.0$ & $70.5 \pm 12.8$ & 0.842 \\
\hline Admission days & $26.5 \pm 5.7$ & $23.9 \pm 7.6$ & 0.077 \\
\hline Period of taking the same dose of warfarin (days) & $15.6 \pm 8.0$ & $11.9 \pm 7.6$ & $0.027^{*}$ \\
\hline Reason for hospitalization & & & 0.274 \\
\hline Cerebral infarction & 43 & 38 & \\
\hline Intracerebral hemorrhage & 0 & 2 & \\
\hline Epidural hemorrhage & 0 & 1 & \\
\hline Subarachnoid hemorrhage & 1 & 0 & \\
\hline Parkinson's disease & 1 & 0 & \\
\hline \multicolumn{4}{|l|}{ Medical history } \\
\hline Hypertension & 37 & 29 & 0.208 \\
\hline Diabetes mellitus & 17 & 9 & 0.110 \\
\hline Dyslipidemia & 13 & 16 & 0.321 \\
\hline Cerebral infarction & 10 & 4 & 0.118 \\
\hline \multicolumn{4}{|l|}{ Indicators of warfarin } \\
\hline Cerebral infarction & 9 & 2 & $0.036^{*}$ \\
\hline Valvular heart disease (non-op) & 1 & 1 & 0.947 \\
\hline Valvular heart disease (op) & 0 & 3 & 0.065 \\
\hline Coronary artery disease & 0 & 2 & 0.134 \\
\hline Atrial fibrillation & 35 & 31 & 0.812 \\
\hline Pulmonary embolism & 1 & 2 & 0.503 \\
\hline Patent foramen ovale & 1 & 0 & 0.337 \\
\hline Aortic dissection & 0 & 1 & 0.292 \\
\hline \multicolumn{4}{|l|}{ Medications } \\
\hline Antiplatelet drugs & 7 & 10 & 0.304 \\
\hline Antihypertensive drugs & 32 & 25 & 0.321 \\
\hline Antidiabetic drugs & 13 & 6 & 0.112 \\
\hline Antidyslipidemic drugs & 14 & 17 & 0.318 \\
\hline Warfarin dose (mg/day) & $3.0 \pm 1.4$ & $3.3 \pm 1.5$ & 0.258 \\
\hline Initial INR (INR) & $2.4 \pm 0.3$ & $2.4 \pm 0.3$ & 0.371 \\
\hline Number of tests of INR & $4.6 \pm 2.5$ & $4.7 \pm 2.8$ & 0.981 \\
\hline \multicolumn{4}{|l|}{ Initial laboratory findings } \\
\hline AST (IU/L) & $23.8 \pm 9.8$ & $23.8 \pm 10.3$ & 0.992 \\
\hline ALT (IU/L) & $22.8 \pm 16.3$ & $24.1 \pm 18.9$ & 0.719 \\
\hline BUN (mg/dL) & $16.5 \pm 6.3$ & $17.6 \pm 6.1$ & 0.470 \\
\hline Creatinine (mg/dL) & $0.89 \pm 0.27$ & $0.86 \pm 0.25$ & 0.561 \\
\hline Platelet $\left(\times 10^{3} / \mu \mathrm{L}\right)$ & $265.9 \pm 96.3$ & $254.6 \pm 81.2$ & 0.559 \\
\hline Hemoglobin (g/dL) & $13.85 \pm 5.23$ & $12.12 \pm 1.61$ & $0.045^{*}$ \\
\hline Hematocrit (\%) & $42.40 \pm 31.94$ & $35.78 \pm 4.39$ & 0.192 \\
\hline
\end{tabular}

TABLE 2: Changes in the international normalized ratio between the herbal and nonherbal groups.

\begin{tabular}{lccc}
\hline & Herbal group $(n=45)$ & Nonherbal group $(n=41)$ & $P$ value \\
\hline INR greater than 3 (\%) & $9(20.0)$ & $16(39.0)$ & 0.052 \\
INR less than 2 (\%) & $27(60.0)$ & $17(41.5)$ & 0.086 \\
INR changed by one or more units (\%) & $12(26.7)$ & $6(14.6)$ & 0.986 \\
INR increased by one or more units (\%) & $7(15.6)$ & $6(14.6)$ & 0.905 \\
INR decreased by one or more units (\%) & $5(11.1)$ & $2.4 \pm 0.4$ & 0.625 \\
Mean INR (mean \pm SD) & $2.3 \pm 0.4$ & $0.032^{*}$ \\
\hline
\end{tabular}

INR: international normalized ratio.

Data are expressed as number or mean \pm standard deviation; ${ }^{*} P<0.05$ was considered significant. Statistical analysis by Pearson’s Chi-square test. 
TABLE 3: Adverse events ratio between the herbal and nonherbal groups.

\begin{tabular}{cccc}
\hline & Herbal group $(n=45)$ & Nonherbal group $(n=41)$ & $P$ value \\
\hline Patient number $(\%)$ & $3(6.7)$ & $2(4.9)$ & 0.723 \\
\hline
\end{tabular}

Data are expressed as number or mean \pm standard deviation; $P<0.05$ was considered significant. Statistical analysis by Pearson’s Chi-square test.

TABLE 4: Frequency of use of herbal medicines taken by patients with an INR less than 2.

\begin{tabular}{|c|c|}
\hline Herbal medicines & Frequency $(\%)$ \\
\hline Glycyrrhiza uralensis Fisch., Poria cocos (Schw.) Wolf & $27(2.1)$ \\
\hline Angelica gigas Nakai, Dioscorea batatas Decne., Scutellaria baicalensis Georgi, & $26(1.1)$ \\
\hline $\begin{array}{l}\text { Cinnamomum loureirii Nees, Zizyphus jujuba var. inermis Rehder, Ledebouriella divaricata (Turcz.) Hiroe, Panax ginseng C. } \\
\text { A. Mey. }\end{array}$ & $24(1.8)$ \\
\hline Liriope platyphylla Wang et Tang, Paeonia obovata Max., Atractylodes japonica Koidz. & $23(1.7)$ \\
\hline Plantago asiatica L., Zingiber officinale Rosc., Cnidium officinale Makino & $22(1.7)$ \\
\hline Bupleurum falcatum Linne & $21(1.6)$ \\
\hline Raphanus sativus L., Apis indica Radoszkowski, Citrus unshiu Markovich & $20(1.5)$ \\
\hline Triticum aestivum $\mathrm{L}$. & $19(1.4)$ \\
\hline Dryobalanops camphora Colebr., Prunus sibirica L., Rehmannia glutinosa (Gaertner) Libosch. & $18(1.4)$ \\
\hline Aurum, Moschus moschiferus L. & $17(1.3)$ \\
\hline Angelica koreanum (Max.) Kitagawa, Pinellia ternata (Thunb.) Breit. & $16(1.2)$ \\
\hline $\begin{array}{l}\text { Glycine max Merr., Ampelopsis japonica (Mak.) Makino, Zizyphus jujuba Mill, Equus asinus L., Gazella subgutturosa Guld., } \\
\text { Schizandra chinensis (Turcz.) Baill., Bos taurus domesticus Gmelin, Typha orientalis Presl }\end{array}$ & $15(1.1)$ \\
\hline Polygala tenuifolia Willd. & $14(1.1)$ \\
\hline Acorus gramineus Sol. ex Aiton, Dimocarpus longan Lour., Gardenia jasminoides J. Ellis & $13(1.0)$ \\
\hline Angelica dahurica Benth. et Hooker f., Amomum villosum Lour., Alisma canaliculatum All. Br. et Bouche & $12(0.9)$ \\
\hline $\begin{array}{l}\text { Rheum palmatum var. palmatum, Hordeum vulgare var. hexastichon Aschers., Inula helenium L., Cimicifuga japonica } \\
\text { Spreng., Atractylodes japonica Koidz., Cyperus rotundus L. }\end{array}$ & $11(0.8)$ \\
\hline $\begin{array}{l}\text { Pueraria thunbergiana Benth., Chrysanthemum indicum L., Citrus aurantium L., Poncirus trifoliata Rafin., Astragalus } \\
\text { membranaceus Bunge, Coptis chinensis Franch }\end{array}$ & $10(0.8)$ \\
\hline $\begin{array}{l}\text { Heracleum hemsleyanum Michx, Ephedra sinica Stapf., Crataegus pinnatifida Bge, Cornus officinalis Sieb. et Zucc., Coix } \\
\text { lacryma-jobi L, Phellodendron amurense Rupr., Magnolia officinalis Rehder et Wilson, Forsythia koreana Nakai }\end{array}$ & $9(0.7)$ \\
\hline $\begin{array}{l}\text { Paeonia suffruticosa Andrews, Polygonum multiflorum Thunb., Phyllostachys nigra var. henonis (Bean.) Stapf, Schizonepeta } \\
\text { tenuifolia (Benth.) Briq. }\end{array}$ & $8(0.6)$ \\
\hline $\begin{array}{l}\text { Castanea crenata S. et Z., Thuja orientalis L., Plantago alata Nakai, Asparagus cochinchinensis Merr., Aconitum carmichaeli } \\
\text { Debx, Areca catechu L. }\end{array}$ & $7(0.5)$ \\
\hline $\begin{array}{l}\text { Trichosanthes kirilowii Maxim., Arisaema amurense Maximowicz, Cannabis sativa L., Asarum sieboldii var. seoulense Nakai, } \\
\text { Perilla frutescens var. acuta Kudo, Achyranthes bidentata Bl., Anthriscus sylvestris var. hirtifructus Hara, Carthamus } \\
\text { tinctorius L., Lonicera japonica Thunb. }\end{array}$ & $6(0.5)$ \\
\hline $\begin{array}{l}\text { Angelica tenuissima Nakai, Pogostemon cablin (Blanco) Benth, Mentha arvensis var. piperascens Malinv, Nelumbo nucifera } \\
\text { Gaertner Polyporus umbellatus (Pers.) Fries }\end{array}$ & $5(0.4)$ \\
\hline $\begin{array}{l}\text { Juncus effusus var. decipiens Buchen., Chaenomeles sinensis Koehne, Akebia quinata var. polyphylla Nak., Morus alba L., } \\
\text { gypsum, Clematis brachyura Max. }\end{array}$ & $4(0.3)$ \\
\hline $\begin{array}{l}\text { Salvia miltiorrhiza Bunge., Sinomenium acutum Rehder et Wils., Amomum cardamomum L., Curcuma zedoaria Rosc., } \\
\text { Scirpus fluviatilis (Torr.) A. Gray, Panax notoginseng (Burk) f. H. Chen, Dendrobium moniliforme (L.) Sw, Lamium album } \\
\text { L., Alpinia oxyphylla Miq., Buthus martensii Karsch, Anemarrhena asphodeloides Bunge, Citrus reticulata Blanco, Talc }\end{array}$ & $3(0.2)$ \\
\hline $\begin{array}{l}\text { Terminalia chebula var. tomentella Kurt., Oryza sativa L., Dianthus chinensis L., Cibotium barometz J. Smith, Capreolus } \\
\text { capreolus ochracea Thomas, Eucommia ulmoides Oliv., Vitex trifolia L., Sinapis alba L., Liquidambar orientalis Mill., Styrax } \\
\text { benzoin Dryand., Acanthopanax seoulense Nakai, Rhinoceros unicornis L., Lindera strychnifolia (Sieb. et Zucc.), Pistacia } \\
\text { lentiscus Joel, Paeonia obovata Max., Eugenia caryophyllata Thunb., Aquilaria agallocha Roxb., Psoralea corylifolia L., Croton } \\
\text { tiglium L., Fritillaria verticillata var. thunbergii (Miq.) Baker, Polygonum bellardi var. effusum Meisn, Piper longum L., } \\
\text { Scrophularia buergeriana Miq. }\end{array}$ & $2(0.2)$ \\
\hline $\begin{array}{l}\text { Alpinia officinarum Hance, Prunus persica (L.) Batsch, mirabilite, Bombyx mori L., Dolichos lablab L., Rubus schizostylus } \\
\text { Lev., Adenophora triphylla var. hirsuta Nakai, Morus alba L., Perilla sikokiana Nakai, Gentiana jamesii Hemsl., Arctium } \\
\text { lappa L., Prunus ishidoyana Nakai, Artemisia scoparia Waldst. et Kit., Polygonum multiflorum Thunb., Uncaria sinensis } \\
\text { (Oliv.) Havil., Lycium barbarum L., Sanguisorba longifolia Bertol., Gentiana macrophylla Pall., Trichosanthes kirilowii } \\
\text { Maxim., Cuscuta chinensis Lam., Taraxacum sinicum Kitag }\end{array}$ & $1(0.1)$ \\
\hline
\end{tabular}


TABLE 5: Frequency of herbal medicines taken by patients with an INR greater than 3.

\begin{tabular}{|c|c|}
\hline Herbal medicines & Frequency $(\%)$ \\
\hline $\begin{array}{l}\text { Glycyrrhiza uralensis Fisch., Angelica gigas Nakai, Zizyphus jujuba var. inermis Rehder, Atractylodes macrocephala Koidz, } \\
\text { Panax ginseng C. A. Mey., Zingiber officinale Rosc. }\end{array}$ & $9(2.3)$ \\
\hline $\begin{array}{l}\text { Platycodon grandiflorum (Jacq.) A. DC., Liriope platyphylla Wang et Tang, Poria cocos (Schw.) Wolf, Paeonia lactiflora } \\
\text { Pallas, Dioscorea batatas Decne., Ligusticum chuanxiong Hort, Scutellaria baicalensis Georgi }\end{array}$ & $8(2.1)$ \\
\hline $\begin{array}{l}\text { Aurum, Glycine max Merr. Saposhnikovia divaricata Schiskin, Ampelopsis japonica (Mak.) Makino, Apis mellifera L., } \\
\text { Moschus moschiferus L., Bupleurum falcatum Linne, Triticum aestivum L., Equus asinus L., Gazella subgutturosa Guld., } \\
\text { Dryobalanops aromatica Gaertn. f., Bos taurus domesticus Gmelin, Cinnamomum cassia Blume, Typha orientalis Presl, } \\
\text { Prunus armeniaca L. var. ansu Maxim. }\end{array}$ & $7(1.8)$ \\
\hline $\begin{array}{l}\text { Raphanus sativus var. hortensis for. acanthiformis Makino, Schizandra chinensis (Turcz.) Baill., Rehmannia glutinosa } \\
\text { (Gaertner) Libosch. }\end{array}$ & $6(1.5)$ \\
\hline $\begin{array}{l}\text { Chrysanthemum morifolium Ramat., Pinellia ternata (Thunb.) Breit., Angelica dahurica Benth. et Hooker f., Zizyphus } \\
\text { jujuba Mill, Dimocarpus longan Lour., Polygala tenuifolia Willd. }\end{array}$ & $5(1.3)$ \\
\hline $\begin{array}{l}\text { Rheum palmatum L., Amomum villosum Lour., Acorus gramineus Sol. ex Aiton, Gardenia jasminoides var. grandiflora } \\
\text { (Lour.) Nakai, Coptis deltoidea C. Y. Cheng et Hsiao, Phellodendron amurense Rupr. }\end{array}$ & $4(1.0)$ \\
\hline $\begin{array}{l}\text { Pueraria thunbergiana Benth., Ostericum koreanum (Max.) Kitagawa, Hordeum vulgare L., Thuja orientalis L., Nelumbo } \\
\text { nucifera Gaertner, Citrus unshiu Markovich, Asparagus cochinchinensis Merr. }\end{array}$ & $3(0.8)$ \\
\hline $\begin{array}{l}\text { Angelica tenuissima Nakai, Trichosanthes kirilowii Maxim., Juncus effusus var. decipiens Buchen., Ephedra sinica Stapf., } \\
\text { Aucklandia lappa Decne, Sinapis alba L., Polygonum multiflorum Thunb., gypsum, Asarum sieboldii Miq., Cimicifuga } \\
\text { heracleifolia Kom., Coix lacryma-jobi var. ma-yuen (Roman.) Stapf, Peucedanum decursivum (Miq.) Maxim., Citrus } \\
\text { aurantium L., Atractylodes lancea (Thunb.) DC., Corydalis ternata Nakai }\end{array}$ & $2(0.5)$ \\
\hline $\begin{array}{l}\text { Castanea crenata S. et Z., Dianthus chinensis L., Lonicera japonica Thunb., Arisaema amurense Maximowicz, Angelica } \\
\text { pubescens for. biserrata Shan et Yuan., Cannabis sativa L., Chaenomeles sinensis Koehne, Paeonia suffruticosa Andrews, } \\
\text { Akebia quinata Decne., Commiphora myrrha Engl., Mentha arvensis var. piperascens Malinv., Sinomenium acutum Rehder } \\
\text { et Wils., Dolichos lablab L., Areca catechu L., Crataegus pinnatifida Bge, Cornus officinalis Sieb. et Zucc., Morus alba L., } \\
\text { Dendrobium loddigesii Rolfe., Perilla frutescens var. acuta Kudo, Forsythia suspensa (Thunb.) Vahl, Lindera strychnifolia } \\
\text { (Sieb. et Zucc.), Gentiana scabra Bunge, Clematis mandshurica Rupr., Lonicera japonica Thunb., Buthus martensii Karsch, } \\
\text { Anemarrhena asphodeloides Bunge, Plantago asiatica L., Aconitum carmichaeli Debx, Citrus unshiu Markovich, Alisma } \\
\text { orientalis (Sam.) Juzep, Polygonum aviculare Linne, Cyperus rotundus L., Scrophularia buergeriana Miq., Schizonepeta } \\
\text { tenuifolia (Benth.) Briq., Carthamus tinctorius L., Talc, Magnolia officinalis Rehder et Wilson }\end{array}$ & $1(0.3)$ \\
\hline
\end{tabular}

were prescribed by experts after medical examination. We described the herbal medicines that had been taken by members of the herbal group in Tables 4-6.

There were three patients in the herbal group and two patients in the nonherbal group that showed adverse events. In the herbal group, one patient showed an INR lower than the therapeutic range, a second showed an INR within the normal therapeutic range, and the third showed an INR higher than the therapeutic range. All patients had bleeding risk factors that were directly associated with adverse events such as frequent tracheostomy suctioning, use of Foley catheter, and history of hematochezia. In the nonherbal group, one patient showed a normal therapeutic INR range with bleeding risk factors, such as the use of a Levin tube and a Foley catheter, and the other patient showed a higher INR than the therapeutic range and had no other bleeding risk factors except warfarin. Regardless of INR value or bleeding risk factors, all of these adverse-event cases involved minor bleeding [20], and there was no statistically significant difference in the adverse event ratio between the two groups. Therefore, we concluded that herbal formulas did not increase bleeding tendency when prescribed by experts. However, warfarin can increase bleeding tendency, so continued monitoring is needed.

The difference in mean INR probably occurred because the nonherbal group had taken a higher warfarin dose than the herbal group, although there was no statistically significant difference in the warfarin dose. Furthermore, the mean INR values of both groups were in the therapeutic range [1], indicating that the mean INR values were not clinically meaningful different.

This survey investigated herbal formulas used in clinics for inpatients taking warfarin, so we considered that the clinical situations were fully reflected and all medicines that had been taken were identified. To our knowledge, this is the first retrospective study of a comparison between a warfarintreated group and a group who took warfarin in conjunction with herbal formulas. We investigated not only mean INR, but also changes in INR and adverse events; therefore, the data will be useful to clinicians and for further studies.

Because our study period was short, we cannot show the long-term effects of herbal formulas on INR, and the data do not represent the influence of herbal formulas on INR that were not within the therapeutic range or the effects of individual herbal medicines. In the future, large-scale, long-term, prospective, and individual herbal medicines studies will be needed.

\section{Conclusion}

In this study, the ratio of INR less than 2 and greater than 3, the ratio of INR that increased or decreased by one or more 
TABLE 6: Frequency of use of herbal medicines taken by the herbal group.

\begin{tabular}{|c|c|}
\hline Herbal medicine & Frequency $(\%)$ \\
\hline Glycyrrhiza glabra L. & $45(2.0)$ \\
\hline Angelica acutiloba Kitag., Dioscorea tenuipes Fr. et Sav., Scutellaria baicalensis Georgi & $49(1.9)$ \\
\hline Thuja orientalis L., Paeonia lactiflora Pallas, Poria cocos (Schw.) Wolf & $41(1.8)$ \\
\hline $\begin{array}{l}\text { Liriope platyphylla Wang et Tang, Atractylodes macrocephala Koidz, Ligusticum wallichii var. officinale Yook, Zingiber } \\
\text { officinale Rosc. }\end{array}$ & $40(1.8)$ \\
\hline $\begin{array}{l}\text { Platycodon grandiflorum (Jacq.) A. DC., Zizyphus jujuba var. inermis Rehder, Ledebouriella divaricata (Turcz.) Hiroe, } \\
\text { Cinnamomum cassia Blume }\end{array}$ & $39(1.7)$ \\
\hline Panax ginseng C. A. Mey. & $38(1.7)$ \\
\hline Bupleurum falcatum Linne & $36(1.6)$ \\
\hline Triticum aestivum L. & $35(1.5)$ \\
\hline Apis indica Radoszkowski & $34(1.5)$ \\
\hline Dryobalanops camphora Colebr. & $33(1.5)$ \\
\hline Raphanus sativus L., Prunus sibirica L. & $32(1.4)$ \\
\hline Aurum, Moschus moschiferus L., Rehmannia glutinosa (Gaertner) Libosch. & $31(1.4)$ \\
\hline Citrus unshiu Markovich & $29(1.3)$ \\
\hline $\begin{array}{l}\text { Glycine max Merr., Pinellia pedatisecta Schott, Ampelopsis japonica (Mak.) Makino, Equus asinus L., Gazella subgutturosa } \\
\text { Guld., Bos taurus domesticus Gmelin, Typha orientalis Presl }\end{array}$ & $28(1.1)$ \\
\hline Zizyphus jujuba Mill, Schizandra chinensis (Turcz.) Baill., Polygala sibirica L. & $25(1.1)$ \\
\hline $\begin{array}{l}\text { Notopterygium forbesii Boiss, Rheum palmatum L., Dimocarpus longan Lour., Gardenia jasminoides var. grandiflora (Lour.) } \\
\text { Nakai }\end{array}$ & $23(1.0)$ \\
\hline Acorus gramineus Sol. ex Aiton, Coptis chinensis Franch & $21(0.9)$ \\
\hline Hordeum vulgare L., Inula helenium L., Amomum villosum Lour., Astragalus membranaceus Bunge & $20(0.9)$ \\
\hline Chrysanthemum indicum L., Alisma orientalis (Sam.) Juzep, Cyperus rotundus L., Phellodendron amurense Rupr. & $19(0.8)$ \\
\hline Pueraria thunbergiana Benth., Cimicifuga heracleifolia Kom. & $18(0.8)$ \\
\hline Atractylodes lancea (Thunb.) DC. & $17(0.7)$ \\
\hline $\begin{array}{l}\text { Angelica pubescens for. biserrata Shan et Yuan., Crataegus pinnatifida Bge, Citrus aurantium L., Magnolia officinalis Rehder } \\
\text { et Wilson }\end{array}$ & $15(0.7)$ \\
\hline Ephedra sinica Stapf., Cornus officinalis Sieb. et Zucc., Lonicera japonica Thunb. & $14(0.6)$ \\
\hline $\begin{array}{l}\text { Paeonia suffruticosa Andrews, Phyllostachys nigra var. henonis (Bean.) Stapf, Poncirus trifoliata Rafin., Plantago alata Nakai, } \\
\text { Schizonepeta tenuifolia (Benth.) Briq. }\end{array}$ & $13(0.6)$ \\
\hline Angelica tenuissima Nakai, Asarum sieboldii Miq., Coix lacryma-jobi L., Asparagus cochinchinensis Merr. & $12(0.5)$ \\
\hline Aconitum koreanum R. Raymond, Polygonum multiflorum Thunb., Nelumbo nucifera Gaertner & $11(0.5)$ \\
\hline Mentha arvensis var. piperascens Malinv. & $10(0.4)$ \\
\hline $\begin{array}{l}\text { Agastache rugosa (Fisch. et Meyer) O. Kuntze, Trichosanthes kirilowii Maxim., Arisaema erubescens (Wall). Schott, Juncus } \\
\text { effusus var. decipiens Buchen., gypsum, Forsythia suspensa (Thunb.) Vahl, Achyranthes bidentata Bl., Peucedanum } \\
\text { decursivum (Miq.) Maxim., Areca catechu L. }\end{array}$ & $9(0.4)$ \\
\hline Castanea crenata S. et Z., Akebia quinata Decne., Perilla frutescens var. acuta Kudo & $8(0.4)$ \\
\hline $\begin{array}{l}\text { Cannabis sativa L., Chaenomeles sinensis Koehne, Aconitum carmichaeli Debx, Clematis mandshurica Rupr., Polyporus } \\
\text { umbellatus (Pers.) Fries, Anemarrhena asphodeloides Bunge, Carthamus tinctorius L., Talc }\end{array}$ & $7(0.3)$ \\
\hline Dianthus chinensis L., Polygonum aviculare Linne & $6(0.3)$ \\
\hline $\begin{array}{l}\text { Vitex rotundifolia L. Fil., Amomum kravanh Pierre ex Gagnep, Curcuma zedoaria Rosc., Scirpus fluviatilis (Torr.) A. Gray, } \\
\text { Morus alba L., Dipsacus asper Wall, Alpinia oxyphylla Miq., Citrus unshiu Markovich }\end{array}$ & $5(0.2)$ \\
\hline $\begin{array}{l}\text { Terminalia chebula var. tomentella Kurt., Salvia miltiorrhiza Bunge., Eucommia ulmoides Oliv., Sinomenium acutum Rehder } \\
\text { et Wils., Panax notoginseng (Burk.) f. H. Chen, Dendrobium loddigesii Rolfe., Liquidambar orientalis Mill., Styrax benzoin } \\
\text { Dryand., Rhinoceros unicornis L., Lindera strychnifolia, Syzygium aromaticum Merr et Perry, Aconitum carmichaeli Debx, } \\
\text { Aconitum ciliare DC., Aquilaria agallocha Roxb., Piper longum L. }\end{array}$ & $4(0.2)$ \\
\hline Cibotium barometz J. Smith, Sinapis alba L., Acanthopanax sessiliflorus, Psoralea corylifolia L., Scrophularia buergeriana Miq. & $3(0.1)$ \\
\hline $\begin{array}{l}\text { Oryza sativa L., Cervus nippon Temminck, mirabilite, Dolichos lablab L., Morus alba L., Perilla frutescens var. acuta Kudo, } \\
\text { Gentiana scabra Bunge, Arctium lappa L., Prunus humilis Bunge, Lycium chinense Mill., Gentiana macrophylla Pall., Cuscuta } \\
\text { chinensis Lam., Croton tiglium L., Fritillaria thunbergii Miq., Taraxacum platycarpum H. Dahlsi, Corydalis ternata Nakai }\end{array}$ & $2(0.1)$ \\
\hline $\begin{array}{l}\text { Alpinia officinarum Hance, Sophora flavescens Ait., Lycium chinense Mill., Prunus persica (L.) Batsch, Commiphora myrrha } \\
\text { Engl., Bombyx mori L., Tribulus terrestris L., Rubus coreanus Miq., Spirodela polyrhiza (L.) Schleid., Adenophora triphylla } \\
\text { var. japonica Hara, Cryptotympana pustulata Fabricius, Prunus mume Sieb. et Zucc., Myristica fragrans Houtt., Artemisia } \\
\text { capillaris Thunb., Uncaria sinensis (Oliv.) Havil., Gastrodia elata Bl., Trichosanthes kirilowii Maxim., Amomum tsaoko } \\
\text { Crevost et Lemaire, Alpinia katsumadai Hayata, Sesamum indicum L., Erodium stephanianum Willd. }\end{array}$ & $1(0.0)$ \\
\hline
\end{tabular}


compared to the initial INR, and the ratio of adverse events were not significantly different between the herbal group and the nonherbal group. It is suggested that concurrent use of prescribed herbal medicine and warfarin may not influence INR value or bleeding tendency compared with conventional therapy.

\section{Conflict of Interests}

No author has any conflict of interests in this work.

\section{References}

[1] M. Kuruvilla and C. Gurk-Turner, "A review of warfarin dosing and monitoring," Proceedings (Baylor University Medical Center), vol. 14, no. 3, pp. 305-306, 2001.

[2] D. J. Greenblatt and L. L. Von Moltke, "Interaction of warfarin with drugs, natural substances, and foods," The Journal of Clinical Pharmacology, vol. 45, no. 2, pp. 127-132, 2005.

[3] P. M. Barnes, B. Bloom, and R. L. Nahin, "Complementary and alternative medicine use among adults and children: United States, 2007," National health Statistics Reports, no. 12, pp. 1-23, 2009.

[4] M. Turfan, A. Tasal, F. Ergun, and M. Ergelen, "A sudden rise in INR due to combination of Tribulus terrestris, Avena sativa, and Panax ginseng (Clavis Panax)," Türk Kardiyoloji Derneği Arşivi, vol. 40, no. 3, pp. 259-261, 2012.

[5] C. A. Rivera, C. L. Ferro, A. J. Bursua, and B. S. Gerber, "Probable interaction between Lycium barbarum (Goji) and warfarin," Pharmacotherapy, vol. 32, no. 3, pp. e50-e53, 2012.

[6] R. L. Page II and J. D. Lawrence, "Potentiation of warfarin by Dong Quai," Pharmacotherapy, vol. 19, no. 7, pp. 870-876, 1999.

[7] S.-H. Lee, Y.-M. Ahn, S.-Y. Ahn, H.-K. Doo, and B.-C. Lee, "Interaction between warfarin and Panax ginseng in ischemic stroke patients," Journal of Alternative and Complementary Medicine, vol. 14, no. 6, pp. 715-721, 2008.

[8] D.-J. Choi, W.-K. Cho, and M.-K. Kim, "An analysis of the interactions between warfarin and Korean traditional herbal medicine in a Korean oriental hospital," European Journal of Integrative Medicine, vol. 6, no. 4, pp. 502-512, 2014.

[9] E. J. Kim, S. H. Lee, L. D. Kim et al., "Influence of herbal medicine coadministration on the INR in stroke patients taking warfarin," Journal of Korean Oriental Medicine, vol. 25, no. 2, pp. 165-172, 2004.

[10] S. H. Lee, Y. S. Kim, C. H. Kang et al., "Retrospective sectional study about the effect of the interaction of herbal medicines and warfarin on prothrombin time(INR) in stroke patients," The Journal of Korean Oriental Internal Medicine, vol. 28, no. 3, pp. 464-472, 2007.

[11] E. J. Kim, J. J. Kim, J. Y. Kim et al., "Influence of herbal medicine coadministration on the INR (international normalized ratio) in patients taking warfarin," The Journal of Korean Oriental Internal Medicine, vol. 24, no. 4, pp. 909-914, 2003.

[12] S. G. Lee and H. H. Ryu, "Herbal medicines effect on coagulation system of ischemic patients," Korean Journal of Oriental Physiology \& Pathology, vol. 18, no. 4, pp. 1213-1217, 2004.

[13] D. H. Kwon, H. J. Kim, M. J. Lee, and M. Y. Song, "Evaluation of interaction and safety in administering herb-medicine with warfarin," Journal of Oriental Rehabilitabion Medicine, vol. 20, no. 2, pp. 175-181, 2010.
[14] W. S. Jung, S. W. Kwon, J. Y. Park, S. W. Park, and S. K. Moon, "Influence of combined administration of herbal complexes and warfarin on international normalized ratio in stroke and anoxic brain damage patients: a retrospective study," European Journal of Integrative Medicine, vol. 4, no. 4, pp. e408-e412, 2012.

[15] S. W. Kwon, J. Y. Park, H. S. Byeon et al., "Influence of herbal medicines which include Ginseng co-administration on the PT INR in patients taking Warfarin: 10 case series," The Journal of Korean Oriental Internal Medicine, vol. 31, no. 3, pp. 675-687, 2010.

[16] S. M. Moran, N. Fitzgerald, M. Pope, M. Madden, and C. J. Vaughan, "Warfarin anticoagulation: a survey of patients' knowledge of their treatment," Irish Journal of Medical Science, vol. 180, no. 4, pp. 819-822, 2011.

[17] A. L. N. Wong and T. Y. K. Chan, "Interaction between warfarin and the herbal product Quilinggao," Annals of Pharmacother$a p y$, vol. 37, no. 6, pp. 836-838, 2003.

[18] H. Leung, A. Hung, A. C. F. Hui, and T. Y. K. Chan, "Warfarin overdose due to the possible effects of Lycium barbarum L.", Food and Chemical Toxicology, vol. 46, no. 5, pp. 1860-1862, 2008.

[19] T. O. Cheng, "Ginseng and other herbal medicines that interact with warfarin," International Journal of Cardiology, vol. 104, no. 2, p. 227, 2005.

[20] S. Schulman and C. Kearon, "Definition of major bleeding in clinical investigations of antihemostatic medicinal products in non-surgical patients," Journal of Thrombosis and Haemostasis, vol. 3, no. 4, pp. 692-694, 2005. 


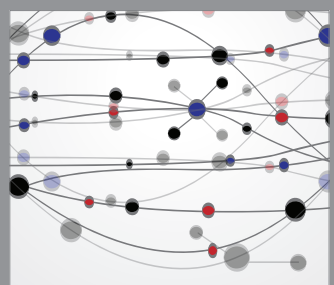

The Scientific World Journal
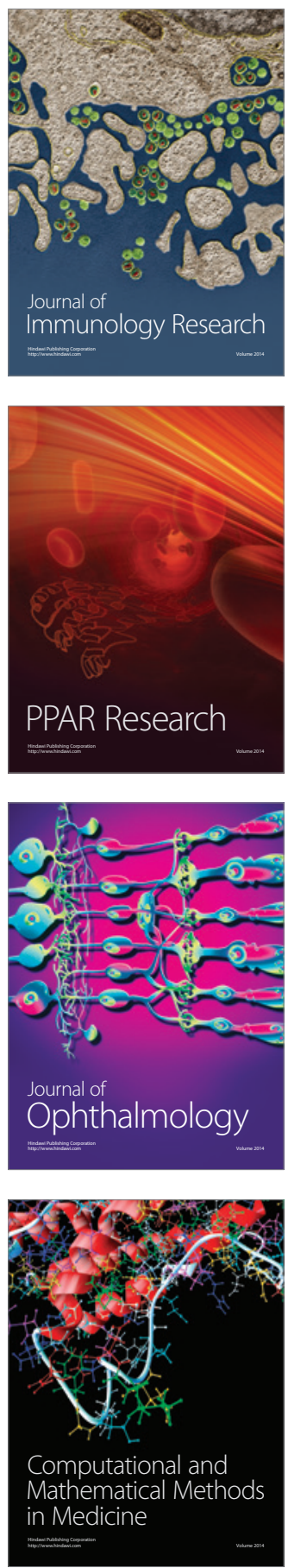

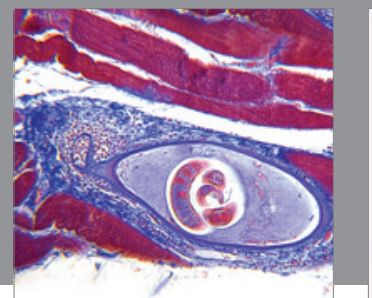

Gastroenterology

Research and Practice
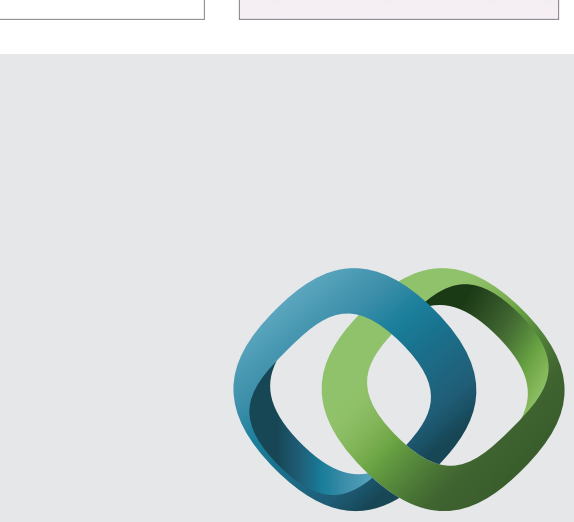

\section{Hindawi}

Submit your manuscripts at

http://www.hindawi.com
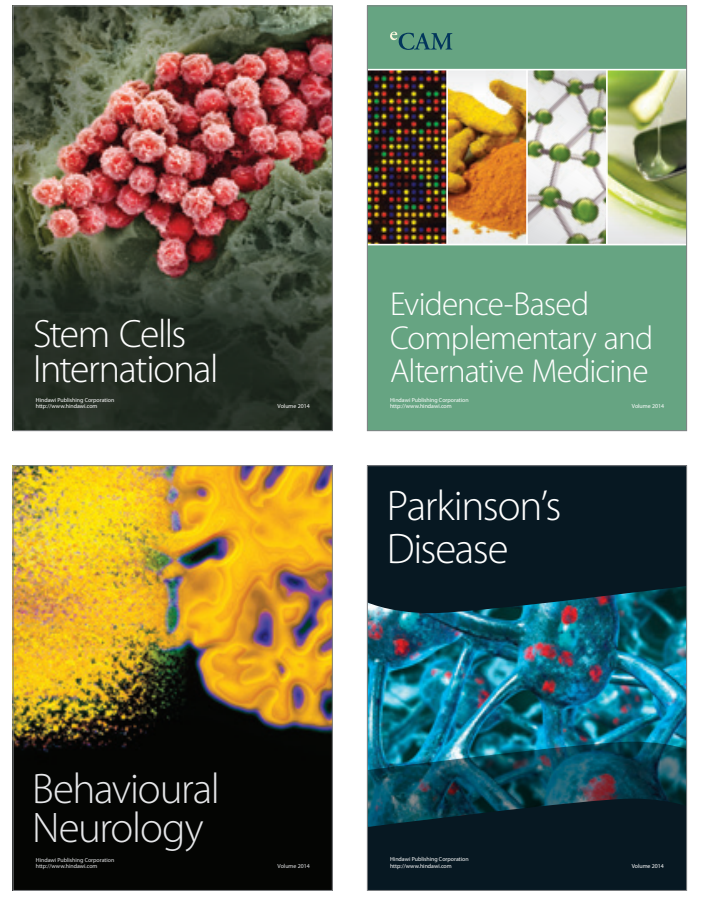
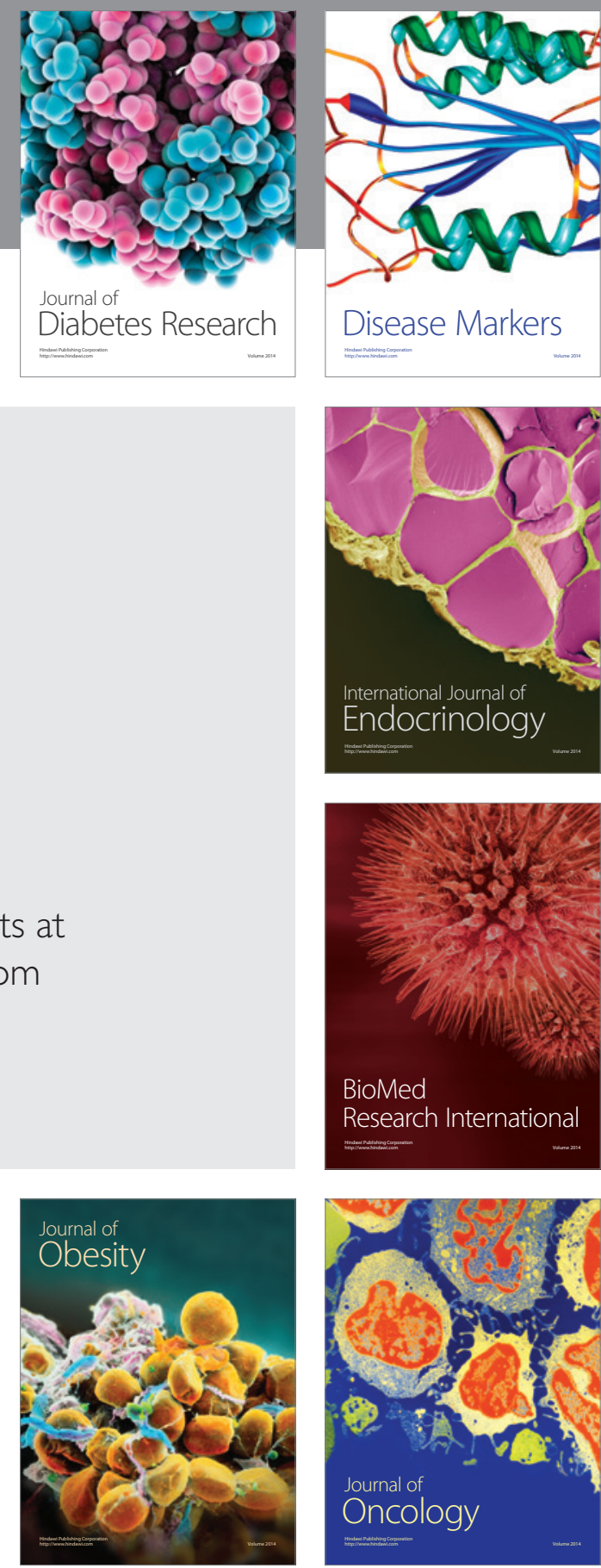

Disease Markers
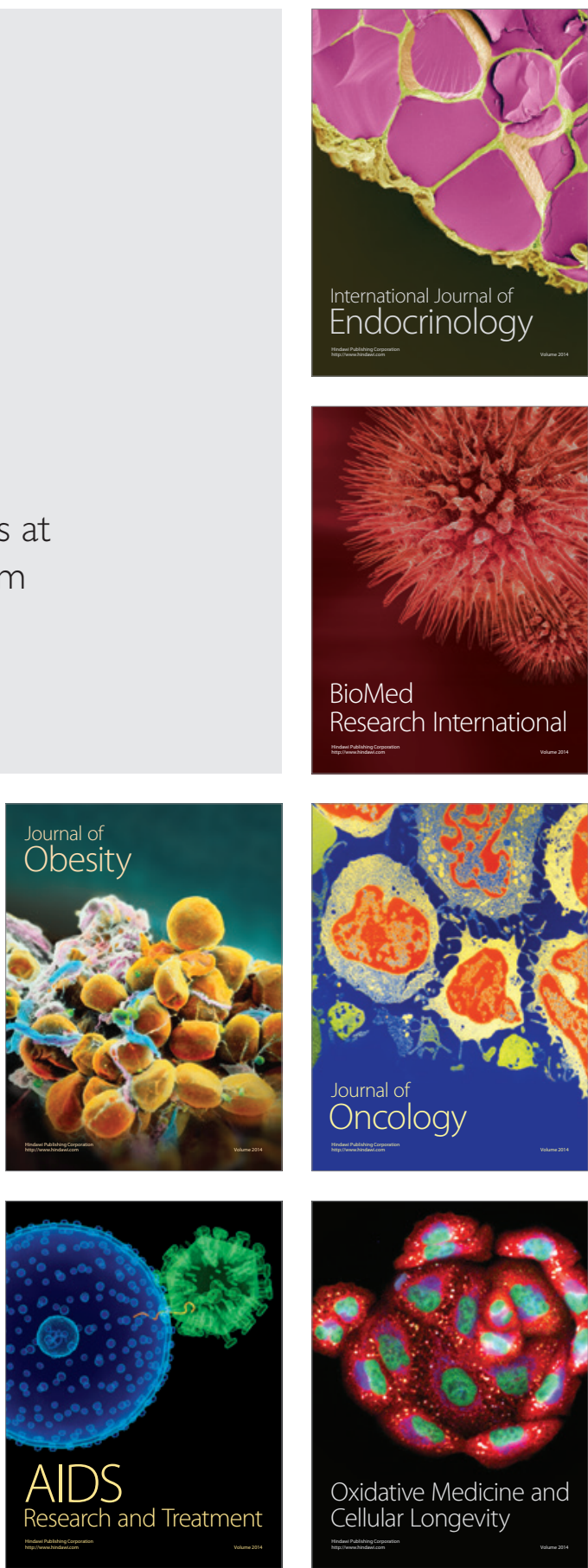